\title{
Problemas éticos en el manejo del dolor. Estudio cualitativo mediante entrevista de reflexión abierta
}

\author{
Ethical problems in the management of pain. Qualitative \\ study through open reflection interview
}

\author{
J. Boceta-Osuna ${ }^{1}$, A. Peiró-Peiró2, F. J. Cevas-Chopitea ${ }^{3}$, L. M. Vidal-Castro ${ }^{4}$, M. S. Acedo-Gutiérrez ${ }^{5}$ \\ y V. Mayoral-Rojals ${ }^{6}$; Grupo de trabajo en Bioética de la SED (BioSED)
}

'Unidad de Cuidados Paliativos, Servicio de Medicina Interna, Hospital Universitario Virgen Macarena. Sevilla, España. ${ }^{2}$ Unidad de Farmacología Clínica, Unidad del Dolor, Hospital General Universitario de Alicante. Alicante, España. ${ }^{3}$ Servicio de Oncología y Cuidados Paliativos, Fundación Rioja Salud. España. ${ }^{4}$ Unidad de Dolor, Servicio de Anestesiología, Hospital Povisa. Vigo, España. ${ }^{5}$ Unidad de Dolor y Cuidados Paliativos, Hospital Universitario 12 de Octubre. Madrid, España. ${ }^{6}$ Unidad de Dolor, Servicio de Anestesiología, Hospital Bellvitge. L'Hospitalet de Llobregat, Barcelona, España.

\section{ABSTRACT}

Introduction: The medical practice in the area of pain associates ethical problems in the care of patients with a disease that causes a functional deterioration, with an uncertain prognosis for their labor reintegration, and a great consumption of family and social resources. After the creation of a working group on bioethics within the Spanish Pain Society (SPS), an attempt is made to analyze these problems.

Objective: To know which are the ethical problems that identify professionals (clinical practice, environment and institutions) which concerns SPS members; encouranging an ethical reflection.

Methodology: Qualitative study, based on a semistructured, open interview, send to members of the SPS ( $n=1035$ ), through electronic access, on 4 bioethical aspects: the problems detected in clinical practice, the problems of the work environment, the problems in the work organizations, and possible suggestions. These are grouped as they refer to the indications (beneficence and non-maleficence), justice (understood as equity), autonomy (information and preferences).

Results: A total of $6 \%$ professionals participated in the interview ( $n=62 / 1035)$. A panel was prepared with the 10 main issues identified. They emphasize the

\section{RESUMEN}

Introducción: La práctica médica en el área del dolor plantea problemas éticos en la atención a pacientes con una enfermedad que provoca un deterioro funcional, con un pronóstico incierto para su reinserción laboral, y gran consumo de recursos familiares y sociales. Tras la creación de un grupo de trabajo en bioética dentro de la Sociedad Española del Dolor (SED) se intenta analizar dichos problemas.

Objetivo: Conocer los problemas éticos relacionados con el manejo del dolor (práctica clínica, entorno e instituciones) que preocupan a los profesionales miembros de la SED, así como fomentar una reflexión ética.

Metodología: Estudio cualitativo, basado en una entrevista semiestructurada, abierta, enviada a los miembros de la SED ( $n=1035)$, mediante acceso electrónico, sobre 4 aspectos bioéticos: los problemas detectados en la práctica clínica, los problemas del entorno de trabajo, los problemas en las organizaciones de trabajo, y posibles sugerencias. Estos se agrupan según se refieran a las indicaciones (beneficencia y no maleficencia), la justicia (entendida como equidad) o la autonomía (información y preferencias).

Resultados: Participaron en la entrevista un $6 \%$ de los profesionales $(n=62 / 1035$ ). Se elaboró un
Boceta-Osuna J, Peiró-Peiró A, Cevas-Chopitea FJ, Vidal-Castro LM, Acedo-Gutiérrez MS, Mayoral-Rojals V; Grupo de trabajo en Bioética de la SED (BioSED). Problemas éticos en el manejo del dolor. Estudio cualitativo mediante entrevista de reflexión abierta. Rev Soc Esp Dolor 2020;27(2):89-96
Recibido: 23-02-2019

Aceptado: 03-02-2020

Correspondencia: Ana Peiró

peiro_ana@gva.es 
uncertainty in the taking of decisions in the therapeutic, the limitation of the therapeutic effort, the conditioning of the sanitary system, the relations with the pharmaceutical industry and the search of the excellence.

Conclusions: This qualitative study allows identifying ethical problems that interest professionals dedicated to pain. It is convenient to confirm and size them through quantitative studies.

Keywords: Professional ethics, pain management, qualitative research, basic principles of bioethics.

\section{INTRODUCCIÓN}

El dolor es un problema de salud global, infravalorado durante décadas, y en España afecta a un $20 \%$ de la población adulta de forma crónica. Pese a que cerca de la mitad de los pacientes reciben algún tratamiento analgésico, el $64 \%$ no logra controlarlo (1). En ellos, la calidad de vida se ha convertido en un indicador de la evolución de su estado de salud, como expresión de su funcionamiento vital en relación con sus objetivos, expectativas, valores e intereses.

La práctica médica en el área del dolor plantea problemas éticos en la atención a pacientes con una enfermedad que provoca un deterioro de la funcionalidad, con un pronóstico incierto para su reinserción a la vida productiva, y con gran consumo de recursos familiares y sociales. Es importante que el paciente pueda participar en la toma de decisiones de una manera racional, y no bajo la influencia del desconocimiento, las ideas irracionales o el miedo $[2,3]$.

La decisión de un paciente adulto y competente de negarse a ser tratado debe ser respetada, en coherencia con sus valores (principio de autonomía). Esto puede entrar en conflicto con valores y deberes del profesional, como el deber de salvaguardar la vida y buscar el bien del paciente (principio de beneficencia), el no cometer delito de omisión (deber de socorro), la obligación de no causar daño (principio de no maleficencia) (4), o el deber de garantizar una accesibilidad equitativa (principio de justicia). Estos y otros problemas interesan y preocupan a los profesionales.

La Sociedad Española del Dolor [SED] cuenta con socios activos multidisciplinares, entre los cuales existe un grupo mayoritario de anestesiólogos que trabajan en unidades de dolor, además de farmacólogos, rehabilitadores, psicólogos, internistas, médicos de familia, fisioterapeutas, enfermeras, neurocirujanos y otros perfiles profesionales. Esta sociedad ha creado un Grupo de Interés y Grupo de Trabajo de Bioética (GT BioSED). Sus objetivos principales son sensibilizar a la sociedad científica y a toda la sociedad sobre los aspectos éticos relacionados con el dolor, promover la formación en bioética e impulsar el desarrollo de trabajos científicos sobre bioética y dolor.

Siguiendo el método científico, la primera tarea del grupo es analizar las cuestiones y problemas éticos que panel con las 10 cuestiones principales identificadas. Destacan la incertidumbre en la toma de decisiones en la terapéutica, la limitación del esfuerzo terapéutico, los condicionamientos del sistema sanitario, las relaciones con la industria farmacéutica y la búsqueda de la excelencia.

Conclusiones: Este estudio cualitativo permite identificar problemas éticos que interesan a los profesionales dedicados al dolor. Es conveniente confirmarlos y dimensionarlos mediante estudios cuantitativos.

Palabras clave: Ética profesional, manejo del dolor, investigación cualitativa, principios básicos de la bioética.

perciben e interesan a los socios. Para investigar cuestiones relacionadas con actitudes, valores y deberes éticos, resulta muy adecuado el enfoque de investigación cualitativa (5). De este modo, la entrevista reflexiva permite poner de manifiesto emociones y pensamientos relacionados con situaciones que vivimos. Hace que podamos profundizar en los juicios que hacemos sobre dichas situaciones y descartar algunos prejuicios. Facilita un conocimiento más profundo de la realidad [6] y hace que la experiencia adquiera un significado más profundo $[7,8)$, que posteriormente podrá transformarse en la práctica $(9,10)$. Cuando la reflexión se expresa por escrito, proporciona información sobre los lugares de trabajo desde donde se escribe, y el carácter y los valores de los participantes (11). La reflexión ética debe ayudar a responder a las necesidades de transformación de los procesos laborales y profesionales.

El objetivo principal de este estudio es conocer los problemas éticos relacionados con el manejo del dolor (en su práctica clínica, su entorno, y las instituciones en las que trabajan, que interesan o preocupan a los profesionales miembros de la SED). Como objetivo secundario pretende fomentar la reflexión sobre los problemas éticos en dichos profesionales interesados en el manejo del dolor.

\section{METODOLOGÍA}

Se ha utilizado un enfoque cualitativo, mediante una entrevista semiestructurada, con preguntas abiertas que invitan a la escritura reflexiva. Se invita a todos los socios de la SED $(n=1035)$ a que participen, a través de un link que les dirige a la encuesta-entrevista. La invitación se acompaña de una breve carta de presentación, y se envía el 9 de septiembre de 2016, mediante un correo electrónico desde la secretaría técnica de la SED. Se da un plazo de 2 semanas para contestarla, y se envía un recordatorio $48 \mathrm{~h}$ antes de finalizar el plazo.

La entrevista consta de 4 preguntas abiertas, y no se dan instrucciones que acoten o cierren el modo de contestarlas. Las preguntas abiertas permiten detectar áreas de interés para los socios de un modo amplio, aunque obliga a un trabajo de síntesis posterior para establecer prioridades. Para esa síntesis agrupamos 
ahora las respuestas a las preguntas en 4 bloques, según hagan referencia a las indicaciones (beneficencia y no maleficencia), la justicia (equidad), la autonomía (información) u otros.

\section{La entrevista semiestructurada}

La entrevista pregunta a los profesionales sobre los problemas éticos en el manejo del dolor, en 3 esferas: (i) problemas que detectan en su práctica clínica, (ii) problemas que observan en su entorno y (iii) problemas que aprecian en las organizaciones en las que trabajan. Además (iv) se solicitan posibles sugerencias al GT BioSED. Son 4 formas distintas de invitar a la reflexión y expresión de los problemas éticos que les interesan. De hecho, la encuesta fue un cuestionario con 4 preguntas abiertas para permitir párrafos y texto libre que después analizaríamos. Las 4 preguntas fueron: 1) En tu práctica cotidiana, ¿qué problemas o cuestiones te generan dudas de tipo ético?; 2) En tu entorno profesional y laboral, ¿qué asuntos crees merecen una reflexión ética?; 3) En la organización en la que trabajas, ¿qué cuestiones éticas deberían tenerse en más consideración?; 4] ¿Tienes alguna sugerencia para el GT BioSED?

Para elaborar el cuestionario se siguieron los siguientes pasos: 1. Diseño de un cuestionario: a diferencia de otras encuestas que se orientan a listar los problemas explorados, planteamos un cuestionario abierto que invite a la reflexión. 2. Ensayo: se remitió el cuestionario de la entrevista a 10 profesionales no incluidos en el estudio, para identificar si se entiende y si los resultados responden al objetivo para el que se confeccionó. 3. Selección de los participantes: no es necesario un muestreo, el propósito no es conseguir representatividad estadística. Los participantes han de tener información que aportar, ser accesibles y estar motivados. 4. Envío del cuestionario de la entrevista a través de un mailing a todos los socios de la SED, con una hoja de información solicitando su participación.

\section{Análisis exploratorio de las respuestas}

Dos investigadores leen por separado las reflexiones enviadas por los participantes, sin un modo predeterminado de clasificarlas. Redactan titulares para resumir los contenidos clave de las reflexiones, y realizan un listado de categorías. Sintetizan los textos largos resaltando el problema ético que mencionan, comprobando las veces que se repite. Desglosan los párrafos que aluden a varios problemas éticos, y registran por separado si hay reiteración de problemas éticos, para facilitar la observación de la saturación de las cuestiones detectadas.

Posteriormente esas síntesis son agrupadas con encabezamientos de mayor orden, bajo los que se unen las que aluden a problemas similares. Se decide agruparlas en 4 epígrafes relacionados con las categorías de la metodología de deliberación ética de Jonsen y los principios éticos. El método de Jonsen invita a analizar los problemas ético-clínicos analizando la situación desde 4 aspectos: el apartado indicaciones tiene que ver con los principios de no maleficencia y beneficencia; el apartado preferencias se relaciona con el principio de autonomía, para lo cual requiere analizar información y capacidad; el apartado calidad de vida se relaciona con el principio de beneficencia; y el de rasgos contextuales aborda sobre todo la justicia equitativa.

La tarea de síntesis y agrupación se realiza independientemente por parte de 2 revisores, y se consensúa con ayuda de un tercer revisor. En total se seleccionaron los 10 problemas éticos más frecuentes, con un mínimo de 2 por cada grupo (indicaciones, justicia, autonomía, otros aspectos]. Este fue el criterio para asignar la importancia de las cuestiones detectadas, aparte del número de veces citadas. Se realizó una reunión del grupo para poner en común los resultados. Al ser preguntas de texto libre, en algunos casos, una misma contestación podía incluir 2 aspectos diferentes según el principio ético. En estos casos, se decidió que se contabilizarían en el apartado más coherente con el resto de la respuesta.

\section{RESULTADOS}

Participaron un $6 \%$ de profesionales de los que recibieron la entrevista $(n=62)$. No se registró edad, género o profesión. Presentan, bajo los epígrafes mencionados, las síntesis de las reflexiones sobre las cuestiones éticas presentes en las reflexiones de los participantes, las que perciben tanto en su práctica cotidiana como en su entorno laboral, en las organizaciones en las que trabajan, o en las sugerencias al grupo investigador. La concordancia entre los investigadores una vez terminado el proceso de clasificación y consenso fue total.

La Tabla I sintetiza las reflexiones que atañen a todos los principios bioéticos. La síntesis de las 10 cuestiones éticas más relevantes [concordancia, fiabilidad, interpretar resultados entre los investigadores] más mencionadas en las reflexiones de los participantes se expone en la Tabla II.

\section{Principios beneficencia/no maleficencia (indicaciones, calidad de vida)}

Se muestra a continuación la síntesis de reflexiones de tipo ético sobre las indicaciones en base a los principios de beneficencia y no maleficencia.

a) En la propia práctica cotidiana: respecto a las técnicas invasivas, 9 comentarios plantearon cuestiones sobre la variabilidad en las indicaciones, la incertidumbre sobre su efectividad, y el conflicto entre intentar aliviar a toda costa el dolor en un paciente frente al deber de ser eficiente. Se plantean la necesidad de protocolos. Preocupa la cuestión de cuándo suspender los tratamientos y qué alternativas plantear para no abandonar al paciente.

Respecto a los fármacos, en 10 comentarios se plantean problemas sobre el uso de opioides: la prescripción y manejo, identificación de malas prácticas, el uso de opioides de liberación rápida con posibilidad de adicción y abuso (que preocupa 
TABLA I

PRINCIPALES PROBLEMAS (CUESTIÓN DISCUTIBLE QUE HAY QUE RESOLVER O EXPLICAR) ÉTICOS MENCIONADOS POR LOS PARTICIPANTES

\begin{tabular}{|l|l|}
\hline \multicolumn{1}{|c|}{ Principio ético } & \multicolumn{1}{c|}{ Problemas éticos } \\
\hline Beneficencia y no maleficencia & $\begin{array}{l}\text { Tratamientos (técnicas, opioides) } \\
\text { Guías y protocolos } \\
\text { Fin de vida: encarnizamiento/deber de socorro }\end{array}$ \\
\hline Justicia, equidad & $\begin{array}{l}\text { Problemas en consulta (medios, listas de espera) } \\
\text { Vulnerabilidad de los pacientes } \\
\text { Relaciones con la industria }\end{array}$ \\
& $\begin{array}{l}\text { Protocolos terapéuticos } \\
\text { Sensibilización a administración/sociedad } \\
\text { Formación }\end{array}$ \\
\hline Autonomía: información, consentimiento & $\begin{array}{l}\text { Consentimiento informado } \\
\text { Vulnerabilidad de los pacientes } \\
\text { Variabilidad interindividual }\end{array}$ \\
\hline Otros & Investigación \\
& Ensayos clínicos \\
& Formación \\
& Realización de un estudio cuantitativo \\
\hline
\end{tabular}

TABLA II

PRINCIPALES PREOCUPACIONES (TEMOR PRODUCIDO POR UN PROBLEMA) ÉTICAS MENCIONADAS POR LOS PARTICIPANTES

\begin{tabular}{|c|c|}
\hline Áreas & Preocupaciones (10 más relevantes] \\
\hline $\begin{array}{l}\text { 1. Indicación de técnicas analgésicas } \\
\text { invasivas }\end{array}$ & $\begin{array}{l}\text { Incertidumbre, escasez de evidencia. Futilidad } \\
\text { Variabilidad y necesidad de protocolos } \\
\text { Equidad en las técnicas caras } \\
\text { Eficiencia vs. compasión en casos concretos }\end{array}$ \\
\hline 2. Indicación de fármacos & $\begin{array}{l}\text { Indicaciones fuera de ficha técnica, variabilidad, uso compasivo, } \\
\text { necesidad de protocolos } \\
\text { Opioides: riesgo de adicciones y problemas a largo plazo }\end{array}$ \\
\hline $\begin{array}{l}\text { 3. Información y consentimiento } \\
\text { informado }\end{array}$ & $\begin{array}{l}\text { Cuánto y cómo informar: información sobre riesgos, asustar vs. } \\
\text { informar, veracidad en el pronóstico vs. transmitir esperanza } \\
\text { Rechazos de tratamientos y sus implicaciones } \\
\text { Valoración de la capacidad, capacidad para qué } \\
\text { Consentimiento por representación } \\
\text { Conspiración de silencio }\end{array}$ \\
\hline $\begin{array}{l}\text { 4. Limitación y adecuación de esfuerzo } \\
\text { terapéutico }\end{array}$ & $\begin{array}{l}\text { Retirada y no inicio de tratamientos, incluidos tratamientos de } \\
\text { soporte vital }\end{array}$ \\
\hline 5. Sedación paliativa & Indicación y consentimiento. Protocolos \\
\hline $\begin{array}{l}\text { 6. Condicionamientos del sistema } \\
\text { sanitario que generan conflicto ético }\end{array}$ & $\begin{array}{l}\text { Sobrecarga de trabajo, falta de tiempo, condiciones laborales que } \\
\text { repercuten en la calidad. Listas de espera. "Recomendación" de } \\
\text { prescribir unos u otros fármacos }\end{array}$ \\
\hline 7. Relaciones con la industria & Influencias, modas, conflicto de intereses \\
\hline 8. Variabilidad en la práctica clínica & $\begin{array}{l}\text { Necesidad de protocolos, reuniones de equipo, excepciones a los } \\
\text { protocolos }\end{array}$ \\
\hline $\begin{array}{l}\text { 9. Búsqueda de la excelencia profesional } \\
\text { y sus limitaciones }\end{array}$ & $\begin{array}{l}\text { Aprendizaje y supervisión de técnicas } \\
\text { Formación y acreditación, calidad } \\
\text { Problemas prácticos que dificultan la excelencia }\end{array}$ \\
\hline $\begin{array}{l}\text { 10. Incertidumbre en la toma de } \\
\text { decisiones }\end{array}$ & Deliberación \\
\hline
\end{tabular}


más en pacientes jóvenes con dolor no oncológico), y las indicaciones de fármacos fuera de ficha técnica y como uso compasivo.

Respecto a la atención a pacientes con enfermedades avanzadas o terminales, se reciben 9 comentarios sobre limitación o adecuación de esfuerzo terapéutico, sedación paliativa, atención al estado vegetativo, dificultades para la analgesia en la demencia, influencia de los familiares, y hasta dónde llegar en la aplicación de técnicas.

b) En el entorno laboral: cinco comentarios mencionan el "primum non nocere". Plantean los problemas de la aplicación de técnicas invasivas que puedan resultar fútiles, en un contexto de insuficiente evidencia, y la necesidad de establecer protocolos unificados que eviten la inequidad y favorezcan la seguridad del paciente. También preocupa la polifarmacia. Se mencionan los errores por confusión entre dolor y sufrimiento, el problema de los pacientes rentistas, la necesidad de revisar y ajustar la dosis a las necesidades, y los conflictos con el posible uso inadecuado de opioides. En 7 comentarios se mencionan problemas sobre final de la vida, limitación de esfuerzo terapéutico y sedación paliativa.

c) Respecto a las organizaciones: seis comentarios reflexionan sobre la promoción de la excelencia profesional, el buen trato, la empatía, el apoyo emocional en la sanidad pública. Preocupa la limitación y adecuación de esfuerzo terapéutico, la posibilidad de obstinación terapéutica y la sedación paliativa.

Como sugerencia para el grupo de bioética se plantean la sensibilización de las organizaciones, y realizar actividades de formación sobre tratamientos fuera de ficha técnica y de uso compasivo, valoración integral y cuidados paliativos.

\section{Principio de justicia (rasgos contextuales]}

a) En la propia práctica cotidiana: seis comentarios mencionan las listas de espera y la presión asistencial, que impiden la buena práctica clínica. Preocupan la priorización de las citas, los pacientes oncológicos, los que residen lejos, y la atención a pacientes fuera de horario. En 5 comentarios se menciona la posible influencia de la industria en el uso de nuevos fármacos y los posibles conflictos de intereses. También preocupa el conflicto ante la indicación de un fármaco o práctica distinta a las recomendadas por el sistema sanitario.

b) En el entorno laboral: nueve comentarios mencionan la necesidad de sensibilización de los profesionales y del sistema sanitario ante el drama del dolor crónico, sus consecuencias y la necesidad de un abordaje integral. Seis comentarios inciden en el problema de las listas de espera y la presión asistencial, con tiempo insuficiente para una atención integral. Cuatro comentarios reflexionan sobre aspectos éticos de las condiciones laborales y el riesgo de burn out. Cinco comentarios sobre la necesidad de formación y gestión del conocimiento plantean, entre otras, la necesidad de ayuda y supervisión de expertos para realizar las técnicas. Cuatro comentarios muestran preocupación por la influencia de la industria farmacéutica y los conflictos de interés.

c) Respecto a las organizaciones: seis comentarios reflexionan sobre la necesidad de coordinación, a nivel regional (planes de dolor), en los hospitales [comités de dolor], entre los distintos servicios y con atención primaria, y dentro de cada servicio o unidad (sesiones, protocolos). Ocho comentarios mencionan las listas de espera y el modo de priorizar la asistencia y establecer el seguimiento ante esa demora. Cinco comentan el problema de los costes de los tratamientos, el conflicto que suponen en la medicina privada y en la sanidad pública. Tres reflexionan sobre el respeto, dignidad y no discriminación de pacientes por lejanía, edad o salud mental, $Y 2$ comentarios mencionan el insuficiente apoyo psicológico.

Preocupa también la necesidad de formación en técnicas y la posibilidad de realizarlas con ayuda experta, y las sustituciones en vacaciones.

Como sugerencias al grupo, 4 comentarios proponen una reflexión ética ante la saturación del sistema sanitario. Tres proponen demandar más recursos materiales y humanos. Seis mencionan la necesidad de actuar según protocolos y guías, evaluar su eficiencia y calidad, y considerar excepciones. Cuatro sugieren que se evalúe el grado de evidencia de las prácticas frecuentes en unidades de dolor y cuidados paliativos. Tres proponen elaborar un código de buenas prácticas en relación con la industria. También proponen enseñar estrategias de autocontrol para separar la presión e influencias externas frente al interés del paciente, mencionando el problema del rechazo de tratamientos por creencias, e insisten en la necesidad de especialización.

\section{Principio de autonomía [preferencias]}

a) En la propia práctica cotidiana: cuatro comentarios reflexionan acerca de la información que se da al paciente sobre las técnicas analgésicas y los fármacos, sus expectativas de eficacia, riesgos y efectos secundarios, y el grado de información a pacientes oncológicos. Seis comentarios se preocupan por la valoración de la competencia y el consentimiento por representación (en menores, incapacidad, ante desacuerdos familiares, etc.).

b) En el entorno laboral: preocupa en 8 comentarios el problema de ocultar información al paciente y su derecho a decidir informando.

c) Respecto a las organizaciones: cuatro comentarios abordan la necesidad de informar a los pacientes y 2 comentan sobre la confidencialidad y privacidad.

Como sugerencias al grupo, 3 comentarios proponen reflexionar sobre la información al paciente en el consentimiento informado. 


\section{DISCUSIÓN}

El método cualitativo utilizado es una variante de entrevista en profundidad, mediante encuesta abierta reflexiva, y sus principales inconvenientes son que el análisis de los resultados resulta laborioso para los investigadores y que no facilita la participación masiva. Aun así, ofrece claras ventajas para investigar no solo sobre los hechos percibidos. Facilita la expresión de las reflexiones de los profesionales sobre su experiencia, y la manifestación de emociones y pensamientos relacionadas con las situaciones vividas, ayudando a identificar los valores en conflicto y los deberes identificados por los profesionales, que son el contenido fundamental que estudia la bioética según explica el profesor Gracia $(12,13)$. Facilita la expresión de las reflexiones de los profesionales sobre su experiencia, y la manifestación de emociones y pensamientos relacionados con las situaciones vividas, ayudando a profundizar en los problemas éticos que plantean dichas situaciones.

Pese al escaso número de respuestas, en torno al $6 \%$ de los socios, la participación resultó suficiente para comprobar una saturación en las cuestiones y problemas detectados, que se repiten en numerosas respuestas enviadas por profesionales expertos y motivados, lo cual demuestran al contestar al cuestionario y en el contenido de las respuestas. No se pretende una representatividad estadística.

Este enfoque cualitativo debería completarse con un nuevo estudio con enfoque cuantitativo, mediante una encuesta cerrada sobre los contenidos detectados en esta primera, puesto que la entrevista de reflexión abierta explora comprender las creencias y los valores del entrevistado, y una metodología de grupo focal aportaría más información, al graduar la importancia de las respuestas en virtud de la frecuencia, evidencia y consistencia de las mismas. Esto ofrecería más rigor en la interpretación de los resultados.

En las cuestiones éticas exploradas se aprecian problemas como la incertidumbre en la toma de decisiones terapéuticas, la limitación del esfuerzo terapéutico, los condicionamientos del sistema sanitario que incluyen, la búsqueda de la excelencia, o las relaciones con la industria farmacéutica. Estos problemas emergentes son similares a los encontrados por otros autores $(14,15)$. En un futuro se categorizará por tipo de dolor lagudo por ejemplo, posquirúrgico, crónico, oncológico o no) puesto que tal vez las cuestiones éticas puedan diferir en su manejo, sobre todo en los casos potencialmente más prevenibles como el dolor agudo posquirúrgico.

Respecto al principio de autonomía, muchos comentarios aluden a problemas relacionados con la información y la capacidad y el consentimiento informado. No se respeta la autonomía del paciente si se considera a priori que este no la posee o está disminuida a causa de su enfermedad, no se toman en cuenta sus opiniones y deseos, y se le ocultan los detalles de su condición olvidando que sus preferencias y valores son primordiales.

Respecto a las indicaciones (principios de beneficencia y no maleficencia), en las técnicas invasivas la variabilidad en las indicaciones plantea un conflicto de justicia (inequidad), que se menciona de diversas formas. La incertidumbre sobre la efectividad de una técnica se ve aumentada por la insuficiente evidencia, y se refleja en los problemas del entorno más que en los de la propia práctica clínica, motivando la reflexión sobre la necesidad de protocolos para evitar hacer daño ["primum non nocere"). También se plantea un conflicto entre beneficencia y justicia, ante la obligación de ser eficientes en aras de la sostenibilidad. La aspiración a la excelencia profesional y la búsqueda de la seguridad del paciente llevan a plantear la necesidad de ayuda y supervisión experta para realizar las técnicas. La preocupación por cuándo suspender los tratamientos ineficaces se acompaña de comentarios sobre el deber de no abandonar al paciente cuando se agota el arsenal terapéutico.

En las indicaciones de fármacos destaca la preocupación por los opioides (uso de opioides en general), la posibilidad de adicción y uso aberrante, y la prescripción fuera de indicación en ficha técnica, especialmente en los nuevos opioides de acción ultrarrápida, que tienen mayor potencial adictivo y su indicación en ficha técnica se restringe a pacientes con dolor de base controlado con opioides. Esta preocupación por los opioides ha sido reflejada en algunos estudios (16), pero otros parecen desmentir ese tópico. En la práctica clínica se recomienda valorar los factores predictivos de comportamiento adictivo como el test de Cage positivo [17].

Serán necesarios estudios controlados sobre posibles indicaciones distintas a las incluidas en ficha técnica, y promover actividades de formación que favorezcan el diálogo y el consenso, y eliminen los prejuicios. No se puede ser beneficente si no se puede aliviar el dolor por falta de una formación teórico-práctica rigurosa y actualizada, y se es maleficente cuando alguien, en lugar de abstenerse de realizar acciones perjudiciales, se abstiene de aliviar el dolor al no proporcionar el tratamiento más adecuado. El alivio del dolor está implícitamente ligado a la calidad de la atención del enfermo, y el profesional tiene el deber ético de intentar hacerlo, con los recursos disponibles y con conocimientos adecuados, y para esto se hace necesaria una buena comunicación con el enfermo (18).

En las indicaciones en enfermedades avanzadas y terminales, las principales cuestiones éticas se plantean respecto a la sedación paliativa y la limitación o adecuación de esfuerzo terapéutico (en un sentido amplio, que incluye no solo medidas de soporte vital, sino también otros tratamientos]. Existe relación entre ambas, pues la limitación de técnicas invasivas puede llevar a considerar el dolor como un síntoma refractario, que es criterio de sedación paliativa. Serán cuestiones sobre las que profundizar en actividades de formación, y varios comentarios plantean la necesidad de sensibilizar a las organizaciones y servicios sobre ellas, así como sobre la necesidad de empatía y respeto.

Respecto al principio de justicia (equidad), las listas de espera y la presión asistencial son los problemas más mencionados, tanto respecto a la práctica clínica de los participantes como en su entorno y en las organizaciones. Preocupa establecer prioridades en las citas, especialmente en los pacientes oncológicos, y hay quejas por la saturación que supone citar a pacientes extra, fuera de horario, en las guardias, etc. con el 
consiguiente riesgo de burn out. Se relaciona la presión asistencial con el insuficiente tiempo para realizar una valoración integral y proporcionar apoyo psicológico. También preocupa el conflicto ante la indicación de fármacos o técnicas distintas de lo recomendado por el sistema sanitario, generalmente con criterios de eficiencia y sostenibilidad, que pueden requerir excepciones justificadas.

Aluden a la justicia numerosos comentarios sobre la necesidad de formación, que favorezca la accesibilidad de los pacientes a los mejores tratamientos. Los programas para el estudio del dolor durante la formación de los profesionales de la salud son insuficientes. No se actúa con justicia si se considera que en los pacientes con dolor difícil ya no queda nada por hacer y no se disponen los recursos necesarios para aliviar su sufrimiento, del mismo modo que se haría con otros tipos de enfermedades. Varias reflexiones se ocupan de la influencia de la industria farmacéutica, que puede condicionar la indicación de tratamientos nuevos y más caros, y los conflictos de interés. Los costes de los tratamientos plantean conflictos éticos en la medicina privada y también en la sanidad pública (sostenibilidad/ beneficencia).

También aluden a la equidad las reflexiones que apuntan a la necesidad de coordinación a nivel regional y local, lo cual se plantea también en otros estudios sobre dolor 18 y en otros campos de la medicina entre distintos servicios y atención primaria y especializada. La principal sugerencia en este aspecto es la reflexión ante la saturación del sistema sanitario, con la necesidad de adecuar y rentabilizar recursos materiales y humanos, actuar según protocolos y guías basadas en la evidencia, y evaluar su eficiencia y calidad.

Respecto a la investigación, preocupa el modo de proponer a los pacientes oncológicos su inclusión en ensayos, y la garantía de que los grupos control reciban al menos el tratamiento estandarizado. Proponen la creación de hojas de información sobre preguntas éticas frecuentes a la hora de diseñar estudios, elaborar un plan mínimo de requisitos éticos para un estudio, una técnica nueva o un cambio de práctica clínica. Proponen completar este estudio con un descriptivo cuantitativo mediante una encuesta con preguntas cerradas, y elaborar una guía de bioética de la SED.

\section{CONCLUSIONES}

Los profesionales dedicados al manejo del dolor en España se preocupan por problemas éticos relacionados con la incertidumbre en las indicaciones, especialmente en cuanto a técnicas invasivas y costosas, y el riesgo/beneficio del uso de ciertos fármacos, sobre todo opioides, y en indicaciones fuera de ficha técnica.

Les interesan los problemas relacionados con la información y la capacidad en el consentimiento informado.

Respecto a las enfermedades avanzadas y terminales, les interpelan la conspiración de silencio y las decisiones de limitación de esfuerzo terapéutico, rechazo de tratamientos y sedación paliativa.

Expresan su preocupación por la equidad y por evitar la variabilidad y discriminación, la búsqueda de la excelencia profesional, y los condicionamientos por las restricciones impuestas por las organizaciones sanitarias, las listas de espera, y la influencia de la industria.

Este primer acercamiento con enfoque cualitativo se debería completar con un estudio descriptivo mediante un cuestionario, para cuya elaboración será útil tener en cuenta estos resultados. Las actividades de formación y sensibilización sobre bioética destinadas a los socios de la SED deberían contemplar estos resultados en el diseño de sus objetivos, contenidos y metodologías docentes.

\section{CONFLICTO DE INTERESES}

Los autores declaran no tener ningún conflicto de intereses.

\section{FUENTES DE FINANCIACIÓN}

Ninguna.

\section{AGRADECIMIENTOS}

Al Prof. Diego Gracia Guillén, que ha impulsado el desarrollo de este grupo de trabajo. A todos los miembros del grupo de interés de bioética de la SED.

\section{DECLARACIONES ÉTICAS}

Los autores declaran que los procedimientos seguidos cumplían los reglamentos del comité ético de investigación clínica responsable de Asociación Médica Mundial y la Declaración de Helsinki. Los autores declaran que en este artículo no aparecen datos de pacientes.

\section{BIBLIOGRAFÍA}

1. Carmona L, Ballina J, Gabriel R, Laffon A; EPISER Study Group. The burden of musculoskeletal diseases in the general population of Spain: results from a national survey. Ann Rheum Dis. 2001;60(11):1040-5. DOI: 10.1136/ ard.60.11.1040.

2. Breivik H, Collett B, Ventafridda V, Cohen R, Gallacher D. Survey of chronic pain in Europe: prevalence, impact on daily life, and treatment. Eur J Pain. 2006;10(4):287-333. DOI: 10.1016/j. ejpain.2005.06.009.

3. Katz $N$. The impact of pain management on quality of life. J Pain Symptom Manage. 2002;24 (1 Suppl):S38-47. DOI: 10.1016/S0885-3924(02)00411-6.

4. Remmers PA, Speer AJ. Clinical strategies in the medical care of Jehovah's witnesses. Am J Med 2006;119(12):1013-8. DOl: 10.1016/j.amjmed.2006.04.016.

5. Grupo de trabajo Sociedad Española del Dolor. Bioética [acceso: 28 Nov 2016]. Disponible en: www.sed.es

6. De Cossart L, Fish D, Hillman K. Clinical reflection: A vital process for supporting the development of wisdom in doctors. Curr Opin Crit Care 2012;18(6):712-7 DOl: 10.1097/ MCC.Ob013e328358e239. 
7. Plack MM, Driscoll M, Blissett S, McKenna R, Plack TP. A method for assessing reflective journal writing. J Allied Health. 2005;34(4):199-208.

8. Mann KV. Reflection: understanding its influence in practice. Med Educ. 2008;42(5):449-51. DOI: 10.1111/j.13652923.2008.03081.x

9. Epstein RM, Hundert EM. Defining and assessing professional competence. JAMA. 2002;287(2):226-35. DOI: 10.1001/jama.287.2.226.

10. Gracey CF, Haidet P, Branch WT, Weissmann P, Kern DE, Mitchell G, et al. Precepting humanism: Strategies for fostering the human dimensions of care in ambulatory settings. Acad Med. 2005;80(1):21-8. DOI: 10.1097/00001888$200501000-00007$.

11. Branch WT Jr, Kern D, Haidet P, Weissmann P, Gracey CF, Mitchell G, et al. Teaching the human dimensions of care in clinical settings. JAMA. 2001;286(9):1067-74. DOI: 10.1001/jama.286.9.1067.

12. Gracia-Guillén D. Teoría y práctica de la deliberación moral. En: Lydia Feito L, Gracia D, Sánchez M, editores. Bioética: el estado de la cuestión. Madrid: Ed. Triacastela; 2011. p. 101-54.
13. Gracia D. Deliberation and consensus. En: Chadwick R, ten Have $\mathrm{H}$, Meslin EM, editores. The SAGE handbook of health care ethics: Core and emerging issues. London: SAGE Publications Ltd; 2011. p.84-94. DOI: 10.4135/ 9781446200971.n9.

14. Anwari JS. Medical ethics and chronic pain management. Saudi Med J. 2008;29(6):921-2.

15. Rich BA, Dubois M. Pain, ethics, and public policy. Pain Med. 2011;12(9):1295-6. DOI: 10.1111/j.1526-4637.2011. 01219.x.

16. Pérez-Castañeda T. Aspectos bioéticos en el manejo del dolor por cáncer [acceso: 12 Mar 2017]. Disponible en: www. cbioetica.org/revista/102/102-0914.pdf

17. Kwon $\mathrm{JH}$, Hui D, Bruera E. A pilot study to define chemical coping in cancer patients using the Delphi method. J Palliat Med. 2015;18(8):703-6.

18. Boceta J, de la Torre A, Samper D, Farto M, Sánchezde la Rosa R. Consensus and controversies in the definition, assessment, treatment and monitoring of BTcP: results of a Delphi study. Clin Transl Oncol. 2016;18(11): 1088-97. 